\title{
AN ALGORITHM FOR MINING ASSOCIATION RULES WITH WEIGHTED MINIMUM SUPPORTS
}

\author{
Yuchiang $\mathrm{Li}^{1}$, Chinchen Chang ${ }^{1,2}$, and Jiehshan Yeh $^{3}$ \\ 'Department of Computer Science and Information Engineering, National Chung Cheng \\ University, Chiayi 621, Taiwan \\ 'Department of Information Engineering and Computer Science, Feng Chia University, \\ Taichung 407, Taiwan \\ ${ }^{3}$ Department of Computer Science and Information Management, Providence University, \\ Taichung 433, Taiwan
}

Abstract: Most existing algorithms employ a uniform minimum support for mining association rules. Nevertheless, each item in a publication database, even each set of items, is exhibited in an individual period. A reasonable minimum support threshold has to be adjusted according to the exhibition period of each $k$-itemset. Accordingly, this paper proposes a new algorithm, called WMS, for mining association rules with weighted minimum supports in publication databases. WMS discovers all frequent itemsets which satisfy their individual requirement of minimum support thresholds. WMS applies the group closure property to prune futile itemsets, to reduce the number of candidates generated, and thus to generate the candidate sets efficiently.

Key words: data mining, association rules, multiple minimum support

\section{INTRODUCTION}

Data mining techniques are typically used to gather hidden but potentially useful information from data in data warehouses. Such techniques have come to constitute an important field of research [6]. Mining association rules is one of the core tasks in solving many data mining problems. The Apriori algorithm [4] is the most famous means of mining association rules. The mining of association rules can be divided into two sub-problems: (1) to find all frequent itemsets that occur more frequently 
than the minimum support threshold, and (2) to use these frequent itemsets to generate association rules. Other efficient methods have been studied to discover frequent itemsets in the past $[1,2,8,12]$.

Existing Apriori-like algorithms assume that all itemsets have a uniform minimum support. In practice, however, some items are important, but have low support. The straightforward approach is to lower the minimum support threshold to find these important but rare items. However, a lower minimum support may yield an excess of useless rules. The method of multiple minimum supports allows users to specify different minimum supports of different items, to discover rules that involve both frequent and rare items [11].

Available mining methods cannot efficiently handle a publication-like database. A publication database is also a transaction database wherein each item contains an individual exhibition period [9]. Two essential problems of the current model are as follows. (1) The exhibition period of each item is neglected, and (2) the support of each item is not evaluated on an equitable basis. For example, Fig. 1 refers to a publication database in a bookstore. Items $\{A\}$ and $\{B\}$ are exhibited from 2000 to the end of 2003. However, item $\{E\}$ is exhibited only in 2003. Classic mining techniques use an identical minimum support threshold and ignore the exhibition period of each item. The PPM algorithm has been proposed to solve this problem [9].

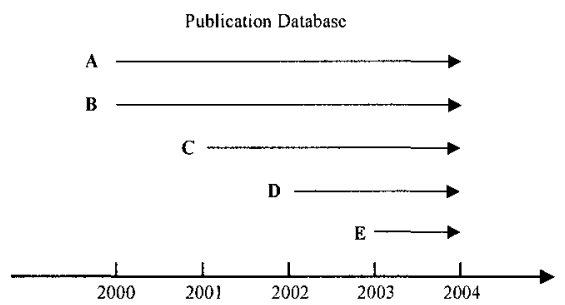

Figure 1. A publication database contains five items

LPMiner employs a linear function to tackle the problem of support that declines with the length of the itemsets. However, the linear function does not take into account the exhibition period of each item. Furthermore, the itemsets with equal length have the same minimum support threshold [13]. The PPM algorithm considers the exhibition period of each item, but it does not deal with the problem of support decrease with the length of itemsets. Moreover, the algorithm assumes that the termination times of all items are identical, but does not reflect on the exhibition period of each $k$-itemset. Therefore, this work proposes a novel weighted minimum supports (WMS) method that considers the individual exhibition period of each $k$-itemset. 
Longer itemsets normally have shorter exhibition periods. Hence, WMS fulfills the requirement that the minimum support thresholds should be lower for longer itemsets. The reasonable exhibition period of a $k$-itemset is defined as the exhibition period shared by its $k$ individual items. WMS also enables the exhibition period of each item to have a distinct cut-off date. This work focuses on generating frequent itemsets in the publication database.

\section{RELATED WORK}

Given a transaction database, the mining of association rules is defined to discover the important rules that relate items. In 1993, Agrawal et al. first defined such mining formally as follows [3]. Let $I=\left\{i_{1}, i_{2}, \ldots, i_{m}\right\}$ represent a set of items (itemset). Each transaction $T$ is also an itemset such that $T \subseteq I$. Let $D B$ be the transaction database, which is a set of transactions. Let $X$ be an itemset; $X$ is contained in $T$ if and only if $X \subseteq T$. An association rule is used in the form $X \Rightarrow Y$, where $X \subseteq I, Y \subseteq I$ and $X \cap Y=\phi$ (For example, $I=\{A$, $B, C, D, E\}, X=\{A, C\}, Y=\{B, D\})$. An rule $X \Rightarrow Y$ has two essential properties, called support and confidence. The support of the rule $X \Rightarrow Y$, defined as $\operatorname{Sup}(X \cup Y)$, is defined as the percentage of the transactions in $D B$ that contain $X \cup Y$. The confidence of the rule $X \Rightarrow Y$, denoted as $\operatorname{Con} f(X \Rightarrow Y)$, is $c \%$ when $c \%$ of the transactions in $D B$ that contain $X$, also contain $Y$. The mathematical expression of confidence is $\operatorname{Conf}(X \Rightarrow Y)=\operatorname{Sup}(X \cup Y) / \operatorname{Sup}(X)$. Given the minimum support (minSup) and minimum confidence (minConf) thresholds for the transaction database $D B$, the mining of association rules discovers all rules whose support, and the confidence in which, are greater than the two minimum thresholds, respectively. An itemset is called a frequent itemset when its support is above the minSup threshold.

Given a user-specified minSup, the Apriori algorithm takes advantage of the downward closure property to filter some infrequent itemsets beforehand [4]. The downward closure property is that any subset of a frequent itemset must be frequent; otherwise the itemset is infrequent. In each pass, Apriori constructs a candidate set of frequent itemsets. Then, the algorithm scans the entire transaction database to determine the frequent itemsets. A candidate $k$ itemset (an itemset with $k$ items) is joined from two arbitrary frequent ( $k-1)$ itemsets, whose first $k-1$ items are identical. If $k \geq 3$, Apriori uses the downward closure property to prune some infrequent itemsets. All remaining $k$-itemsets constitute candidate $k$-itemsets. The process is repeated until no candidate can be generated. 
Apriori employs a single minimum support threshold for whole transaction database. In reality, the minimum support may not be uniform. In 1998, Cai et al. developed the notion of the multiple minimum support thresholds to reflect the importance of each item [5]. In 1999, Liu et al. described an algorithm that exhibits the sorted closure property to solve the problem that the downward closure characteristic cannot be employed in the multiple minimum supports scenario [11].

Example 2.1 Consider the three items $\{A\},\{B\}$, and $\{C\}$ in a database. The individual minimum support threshold of each item is as follows.

$$
\operatorname{minSup}(A)=5 \%, \quad \operatorname{minSup}(B)=10 \%, \quad \text { and } \operatorname{minSup}(C)=15 \% .
$$

The minSup threshold of an itemset is defined as the lowest support value among all items in the itemset. If the support of the itemset $\{B, C\}$ is $8 \%$, then it is under both $\min \operatorname{Sup}(A)$ and $\operatorname{minSup}(B) .\{B, C\}$ is discarded because of downward closure. However, itemset $\{A, B, C\}$ may be frequent because $\min \operatorname{Sup}(A)$ is $5 \%$. The sorted closure property is used to solve the problem by sorting in order of ascending minimum support.

In 2000, Wang et al. presented a "pushing support constraints" method to "push" support constraints into the generation of the Apriori itemset to conserve the essence of Apriori and determine the minimum support for each itemset durning running time [15]. Recent research has developed numerous other mining association rules with multiple minimum supports $[7,9,10,13$, 14]. In this work, the WMS algorithm is also a variant of mining association rules with multiple minimum supports.

\section{WEIGHTED MINIMUM SUPPORTS (WMS)}

The main idea behind the previous multiple minimum supports techniques is the assigning of an individual minimum support threshold to each item. Although the techniques can yield interesting itemsets with low support, they do not account for the lower support threshold of long itemsets. Short itemsets tend to be interesting when they have high support, whereas long itemsets may be of interest but occur relatively infrequently. To solve this problem, the LPMiner algorithm incorporates a support-constraining function, which reduces the support threshold as the length of the itemset increases. However, the minimum support thresholds of equally long itemsets are identical in LPMiner. This study proposes the Weighted Minimum Supports (WMS) algorithm, in which for each itemset, the minimum support is individually weighted, according to its period of exhibition. 


\subsection{Common Exhibition Period}

In a publication database, the exhibition period of each individual itemset may be different, so measurements based on the assumption that all itemsets have an identical "life cycle" are biased. In this work, the exhibition period of each itemset is defined as a span of time granularities. Let a transaction database $D B$ logically be divided into $n$ partitions of unit time granularity (month, quarter or year, for example). The symbol $d b^{t_{1,} t_{2}}$ indicates the continuous portion of the database from partition $P_{t_{1}}$ to partition $P_{t_{2}}$, where $t_{2}$ $\geq t_{1} \geq 1$ and $t_{1}, t_{2} \in N$. A $k$-itemset $I=\left\{i_{1}, i_{2}, \ldots, i_{k}\right\}^{t_{1}, t_{2}}$ denotes that the exhibition period of $\left\{i_{1}, i_{2}, \ldots, i_{k}\right\}$ is from $P_{t_{1}}$ to $P_{t_{2}}$. For the entire database, given a minSup, the weighted minimum support (WminSup) threshold of each 1-itemset is $\min S u p^{*}\left|d b^{t_{1, t}}\right| /|D B|$, where $\left|d b^{t_{1, t_{2}}}\right|$ indicates the number of transactions of $d b^{t_{1}, t_{2}}$ and $|D B|$ represents the number of transactions of $D B$. The weighted minimum support threshold of a $k$-itemset is derived from the common exhibition period of all $k$ items in the $k$-itemset. Consider the $k 1$ itemsets $\left\{i_{1}\right\}^{t_{11}, t_{12}},\left\{i_{2}\right\}^{t_{21}, t_{22}}, \ldots,\left\{i_{k}\right\}^{t_{k}, t_{k 2}}$; the start time of the exhibition of the $k$-itemset $\left\{i_{1}, i_{2}, \ldots, i_{k}\right\}$ is $t_{1}=\max \left\{t_{11}, t_{21}, \ldots, t_{k 1}\right\}$ and the end time is $t_{2}=$ $\min \left\{t_{12}, t_{12}, \ldots, t_{k 2}\right\}$. The period in which these $k$ individual items occur at the same time is from $t_{1}$ to $t_{2}$. If $t_{2}<t_{1}$, then the $k$-itemset does not appear in $D B$.

Example 3.1 Consider a database that contains five individual items. Figure 2 illustrates the exhibition periods of these five 1-itemsets. The common exhibition period of $\{B\}^{2000,2001},\{C\}^{2001,2003}$ and $\{D\}^{2001,2002}$ is the exhibition period of $\{B, C, D\}$. Therefore, the start time of $\{B, C, D\}$ is $\max \{2000$, $2001,2001\}=2001$ and the end time is $\min \{2001,2003,2002\}=2001 .\{B$, $C, D\}^{2001,2001}$ denotes that the exhibition period of $\{B, C, D\}$ is one year, 2001.

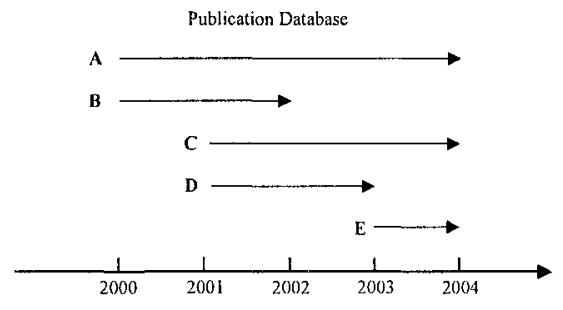

Figure 2. Exhibition period of each 1-itemset

The duration of the exhibition period of an itemset is turned into the minimum support weight. Users can effectively express various minimum support requirements for different rules, in light of the individual weight of each itemset. The use of weighted minimum supports can enable itemsets 
with long exhibition periods to have higher minimum supports and those short exhibition periods itemsets to have lower minimum supports.

\subsection{Candidate Generation}

In [11], Liu et al. used the sorted closure property to solve the problem of mining with multiple minimum supports. However, in this work, the sorted closure property does not be applied.

Example 3.2 Consider three items $\{A\},\{B\}$ and $\{C\}$ in a transaction database, with exhibition periods $\{A\}^{1,4},\{B\}^{1,3}$, and $\{C\}^{3,4}$, respectively. The distributions of the records in the database stretch over four time units. Assume that all partitions include the same number of transactions. The minimum support for the entire database is $40 \%$. Thus, $\operatorname{WminSup}(A)=40 \%$, $W \operatorname{minSup}(B)=30 \%, W \operatorname{minSup}(C)=20 \%$ and $\operatorname{WminSup}(B C)=10 \%$. If the support of itemset $\{B\}=15 \%$, then the itemset is discarded using sorted closure. The occurrence count is below the minimum of all 1-itemset WminSup values, so the potentially frequent itemset $\{B C\}^{2,2}$ is pruned by using the sorted closure property.

A publication database is logically divided into $n$ partitions with unit time granularity. WminSup of each partition can be derived easily from the userdefined minSup. The minimum WminSup is the minimum among all of these WminSups. If the percentage of occurrences of a $k$-itemset is below the minimum WminSup, then all of its supersets must be infrequent. Hence, the $k$-itemset can be pruned. A superset of a $k$-itemset becomes a frequent itemset if the number of supports of the $k$-itemset exceeds the minimum WminSup. Sorted closure is irrelevant to WMS. Too many candidate itemsets will be generated if the minimum WminSup is the only pruned threshold. Therefore, this study describes another property, called the Group Closure Property (GCP), used to prune the number of candidate itemsets and generate the candidate set efficiently.

\section{Group Closure Property (GCP)}

Consider a database with $n$ grains of time. The total number of distinct continuous exhibition periods is $n(n+1) / 2$. Accordingly, each $k$-itemset can be assigned to one of the $n(n+1) / 2$ groups such that each $k$-itemset in the same group has an identical exhibition period. That is, each $k$-itemset in the same group has an identical WminSup. After the $k$-th pass, WMS employs the minimum WminSup value to delete useless candidates. The remaining $k$ itemsets form a set $R C_{k}$. The set $C_{k+1}$ is generated from $R C_{k}$. Let $\operatorname{WminSup}\left(g_{i}\right)$ 
denote the WminSup value of the group $g_{\text {, }}$, and let $W \operatorname{minSup}\left(g_{i} \cap g_{j}\right)$ denote the WminSup value of the common exhibition period of the two groups $g_{i}$ and $g_{j}$, the generation of $C_{k+1}$ proceeds as follows.

1. Sort the groups in order of descending WminSup. $G r$ (first) denotes the set of groups which is sorted, where $\operatorname{Gr}($ first $)=\left\{g_{1}, g_{2}, \ldots, g_{n(n+1) / 2}\right\}$. Select a group $g_{i} \in G r$ (first) in order.

2. Compute the common exhibition period of $g_{i}$ with each other group $g_{j}$, where $i<$ $j$. WMS sorts other groups in order of increasing $\operatorname{WminSup}\left(g_{i} \cap g_{j}\right)$ value; it discards groups that do not share common exhibition period. $G r$ (second) represents the set of groups which is sorted.

3. Select a group $g_{j} \in G r$ (second) in order.

4. As required by the $\operatorname{WminSup}\left(g_{i} \cap g_{j}\right)$, delete the $k$-itemsets in $g_{i}$ whose occurrence counts are smaller than $W \operatorname{minSup}\left(g_{i} \cap g_{j}\right)$.

5. Join each $k$-itemset in $g_{i}$ with each $k$-itemset in $g_{j}$, whose occurrence counts are above the $\operatorname{WminSup}\left(g_{i} \cap g_{j}\right)$ requirement, to generate candidate $(k+1)$-itemsets.

6. Select the next $g_{j}$ and return to Step 4 until all groups have been chosen.

7. Delete all infrequent $k$-itemsets in $g_{i}$. Output frequent $k$-itemsets. Join each frequent $k$-itemset in $g_{i}$ with each other to generate $(k+1)$-itemsets.

8. Select the next $g_{i}$ and return to Step 2 until all groups have been selected.

In Step 4, if the number of occurrences of the $k$-itemset in $g_{i}$ is smaller than $W \operatorname{minSup}\left(g_{i} \cap g_{j}\right)$, then any superset of the $k$-itemset that has not yet be generated must be infrequent, because $G r$ (second) is sorted in order of increasing $W \operatorname{minSup}\left(g_{i} \cap g_{j}\right)$. In Step 5, the candidate $(k+1)$-itemset is not produced if the support value is under the $\operatorname{WminSup}\left(g_{i} \cap g_{j}\right)$ threshold. The method of joining artibrary two candidates is the same as that in Apriori. In Step 7 , all $k$-itemsets which are in the same group exhibit the downward closure property.

Example 3.3 Consider a database in which the distribution of transactions stretches over four time grains. Assume that each partition contains the same number of transactions. The minimum support for the entire database is $40 \%$. Let $g^{t_{1}, r_{2}}$ expresses the group whose exhibition period is from $t_{1}$ to $t_{2}$. The first column in Table 1 lists the ten groups in the $G r$ (first) sequence from top to bottom. The second column presents the corresponding Gr(second) sequences. Assume that the support value of itemset $\{A, B\}^{1,4}$ is $15 \%$. First, $\{A, B\}$ is joined with other 2-itemsets that in the group $g^{1,1}$ to generate the 3itemset candidates. According to the group order $g^{2,2}, g^{3,3}, \ldots, g^{2,4},\{A, B\}$ joins with other 2 -itemsets until the process arrives at group $g^{1,2}$. Because, WminSup $\left(g^{1,4} n g^{1,2}\right)=20 \%$ exceeds $15 \%$, so $\{A, B\}$ is deleted from $\mathrm{g}^{1,4}$ after the group $\mathrm{g}^{1,2}$ is selected.

The pseudo-code for generating candidates is as follows. 
Procedure candidate-gen $\left(R C_{k-1}\right)$

$01 G r($ first $)=\operatorname{sort}\left(G, W \operatorname{minSup}\left(g_{i} \in G\right)\right) ; / / G$ is the set of all groups

02 for each group $g_{i} \in G r($ first $)$ do

$03 G=\left\{\mathrm{g}_{j} \in G r(\right.$ first $\left.) \mid i<j\right\}$;

04 for each $g_{j} \in G$ do

$05 \quad$ if $W \operatorname{minSup}\left(g_{i} \cap g_{j}\right)=0$;

06 delete $g_{j}$; endif endfor

$07 \quad G r($ second $)=\operatorname{sort}\left(G, W \operatorname{minSup}\left(g_{i} \cap g_{i}\right) \neq 0\right)$;

08 for each $c_{i} \in g_{i}$ do

09 if $\mathrm{c}_{i}$. count $<$ WminSup $\left(g_{i} \cap g_{j}\right)$

10 delete $c_{i}$; endif

11 for each $c_{j} \in g_{j}$ do

12 if $\mathrm{c}_{j}$.count $<$ WminSup $\left(g_{i} \cap g_{j}\right)$

13 next; endif

$14 \quad$ apriori-join $\left(c_{i}, c_{j}\right)$;

15 endfor

16 endfor

17 for each $c_{i} \in g_{i}$ do

18 if $\mathrm{c}_{i}$. count $<\operatorname{WminSup}\left(g_{j}\right)$

19 delete $c_{i}$; endif endfor

20 for each $c_{i} \in g_{i}$ do

21 for each $\left(c_{j} \neq c_{i}\right) \in g_{i}$ do

22

23

24 endfor apriori-join $\left(c_{i}, c_{j}\right)$; endfor

endfor

Table 1. Example of the Gr(first) sequence and the Gr(second) sequence

\begin{tabular}{ll}
\hline Gr(first) & Gr(second) sequence, in order of increasing WminSup $\left(g_{i} \cap g_{j}\right)$ \\
\hline $\mathrm{g}_{1}=\mathrm{g}^{1,4}$ & $\left\{\mathrm{~g}^{1,1}, \mathrm{~g}^{2,2}, \mathrm{~g}^{3,3}, \mathrm{~g}^{4,4}, \mathrm{~g}^{1,2}, \mathrm{~g}^{2,3}, \mathrm{~g}^{3,4}, \mathrm{~g}^{1,3}, \mathrm{~g}^{2,4}\right\}$ \\
$\mathrm{g}_{2}=\mathrm{g}^{1,3}$ & $\left\{\mathrm{~g}^{1,1}, \mathrm{~g}^{2,2}, \mathrm{~g}^{3,3}, \mathrm{~g}^{3,4}, \mathrm{~g}^{1,2}, \mathrm{~g}^{2,3}, \mathrm{~g}^{2,4}\right\}$ \\
$\mathrm{g}_{3}=\mathrm{g}^{2,4}$ & $\left\{\mathrm{~g}^{2,2}, \mathrm{~g}^{3,3}, \mathrm{~g}^{4,4}, \mathrm{~g}^{1,2}, \mathrm{~g}^{2,3}, \mathrm{~g}^{3,4}\right\}$ \\
$\mathrm{g}_{4}=\mathrm{g}^{1,2}$ & $\left\{\mathrm{~g}^{1,1}, \mathrm{~g}^{2,2}, \mathrm{~g}^{2,3}\right\}$ \\
$\mathrm{g}_{5}=\mathrm{g}^{2,3}$ & $\left\{\mathrm{~g}^{2,2}, \mathrm{~g}^{3,3}, \mathrm{~g}^{3,4}\right\}$ \\
$\mathrm{g}_{6}=\mathrm{g}^{3,4}$ & $\left\{\mathrm{~g}^{3,3}, \mathrm{~g}^{4,4}\right\}$ \\
$\mathrm{g}_{7}=\mathrm{g}^{1,1}$ & None \\
$\mathrm{g}_{8}=\mathrm{g}^{2,2}$ & None \\
$\mathrm{g}_{9}=\mathrm{g}^{3,3}$ & None \\
$\mathrm{g}_{10}=\mathrm{g}^{4,4}$ & None \\
\hline
\end{tabular}

In Lines 14 and 22, the join function is the same as the join step in Apriori. This function joins two distinct $(k-1)$-itemsets whose first $k-2$ items are identical. Lines 8 to 16 generate candidate $k$-itemsets when arbitrary two $(k-1)$-itemsets are in different groups, whereas from Lines 17 to 23 relate to the case in which all (k-1)-itemsets appear in the same group. In Line 22, although the itemsets in the same group satisfy the property of download closure, the exhibition periods of the subset of a frequent $k$-itemset may be different with the $k$-itemset. Thus, omit the pruning step following the joining step. The candidate generation function deletes in advance $(k-1)$ itemsets whose supersets must be infrequent. Then, the candidate $k$-itemsets 
are generated. The pruning step is implemented only before the joining step, unlike in Apriori, whose pruning step is implemented only after the joining step. Lines 9-10, 12-13 and 18-19 are the pruning steps.

\subsection{Weighted Minimum Supports}

The WMS algorithm is based on a level-wise technique like Apriori. WMS passes over the database $k$ or $k+1$ times to generate all frequent $k$ itemsets. In the first pass, each item is a candidate 1-itemset. WMS scans the database to count the supports of each candidate 1-itemset and determines whether these 1-itemsets are frequent. If the support values of the 1-itemsets are greater than or equal to the minimum WminSup, then these 1-itemsets are added to the set $R C_{1}$. In the pass $k, C_{k}$ is generated from $R C_{k-1}$; then WMS scans the database to count the supports of each candidate $k$-itemset and determines whether they are frequent. The WMS algorithm pseudo-code is stated as follows.

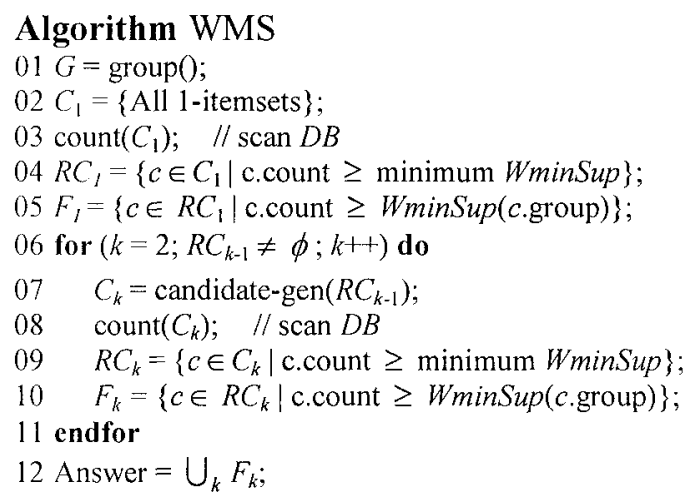

In Line 1, all itemsets are assigned to their corresponding groups, according to the distinct exhibition period of the itemsets. In Line 7, the set $R C_{k-1}$ is used to generate candidate $k$-itemsets. WMS exploits the group closure property to prune some useless candidates and generate the candidate $k$-itemsets. Lines 3 and 8 scan the database and count the support value of the candidate $k$-itemsets.

\section{CONCLUSIONS}

In a publication database, each itemset has an individual exhibition period. Mining association rules on publication databases with a uniform minimum support is unpractical. The reasonable exhibition period of a $k$ itemset is the common exhibition period of its $k$ individual items. The sensible minimum supports of distinct $k$-itemsets should be adjusted in the 
light of their exhibition periods. This investigation introduced a novel approach, WMS, for mining association rules with weighted minimum supports that consider the exhibition period of each itemset in a publication database. WMS also accounts for the distinct end time of the exhibition period of each item. The proposed method mines frequent itemsets whose percentage of occurrences exceeds the individual WminSup threshold. WMS eliminates some useless itemsets using the group closure property to avoid generating an excess of candidates. In the future, we will consider the extension of the WMS algorithm to multiple level association rules.

\section{REFERENCES}

11] R. C. Agarwal, et al. A tree projection algorithm for generation of frequent itemsets. Journal of Parallel and Distributed Computing, 2000, 61(3): 350 361

[2] C. C. Aggarwal and P. S. Yu, Mining associations with the collective strength approach. IEEE Trans. Knowledge and Data Engineering, 2001, 13(6): 863 873

[3] R. Agrawal, et al. Mining association rules between sets of items in large databases. 1993 ACM SIGMOD Intl. Conf. on Management of Data, Washington: 207 216

[4] R. Agrawal and R. Srikant, Fast algorithms for mining association rules. 20th Intl. Conf. on Very Large Data Bases, 1994, Santiago: 487 499.

[5] C. H. Cai, et al. Mining association rules with weighted items. 1998 Intl. Database Engineering and Applications Symposium, Cardiff: $68 \sim 77$

[6] M. S. Chen, et al. Data mining: An overview from a database perspective. IEEE Trans. Knowledge Data Engineering, 1996, 8(6): 866 883

[7] F. L. Chung and C. L. Lui, A post-analysis framework for mining generalized association rules with multiple minimum supports. 6th ACM SIGKDD Intl. Conf. on Knowledge Discovery and Data Mining Workshop on Post-Processing in Machine Learning and Data Mining, 2000, Boston: 9 14

[8] J. Han, et al. Mining frequent patterns without candidate generation. 2000 ACM-SIGMOD Intl. Conf. on Management of Data, Dallas: 1 12

[9] C. H. Lee, et al. Progressive partition miner: An efficient algorithm for mining general temporal association rules. IEEE Trans. Knowledge Data Engineering, 2003, 15(4): 1004 - 1017

[10] C. L. Liu and F. L. Chung, Discovery of generalized association rules with multiple minimum supports. 4th European Conf. on Principles of Data Mining and Knowledge Discovery, 2000, Lyon: 510 515

[11] B. Liu, et al. Mining association rules with multiple minimum support. 1999 ACM SIGKDD Intl. Conf. on Knowledge Discovery and Data Mining, San Diego: 337 341

[12] J. S. Park et al. An effective hash-based algorithm for mining association rules. 1995 ACM-SIGMOD Intl. Conf. on Management of Data, San Jose: 175 186

[13] M. Seno and Karypis, LPMiner: An algorithm for finding frequent itemsets using lengthdecreasing support constraint. 2001 IEEE Intl. Conf. on Data Mining, San Jose: 505 512

[14] M. C. Tseng, et al. Maintenance of generalized association rules with multiple minimum support. Joint 9th IFSA World Congress and 20th NAFIPS Intl. Conf., 2001, Vancouver: 1294 1299

[15] K. Wang, et al. Pushing support constraints into association rules mining. IEEE Trans. Knowledge Data Engineering, 2003, 15(3): 642 658 